\title{
Hypersentivity reactions to COVID-19 vaccines: A case of Eosinophilic pneumonia following Sinovac/CoronaVac vaccination
}

\section{Abstract}

Hypersensitivity reactions has been reported with COVID-19 vaccines A sute eosinophilic pneumonia has not been reported yet after Sinovac/CoronaVac vaccin॰. 4\% j-yearold woman presented with maculopapular rash, cough and dyspi.e $\iota$ following Sinovac/CoronaVac injection. The complete blood count (CBC) indicated oos nophilia and further evaluation of the eosinophilia with $\mathrm{CT}$ and bronchoscopy confirmea , diagnosis of acute eosinophilic pneumonia. After methylprednisolone therapy, her rash $\cdot{ }^{\circ} s^{\prime} /$ lved with marked improvement of the dyspnea. She is still on treatment and on the foin up period, we plan to continue steroid treatment at least 3 months.

Keywords: COVID-19, COVID-19 vaccines, Si` ovac/CoronaVac, SARSCoV-2, eosinophiles, eosinophilic pneumonia, rash.

\section{Introduction}

Severe acute respiratory syndrome con navirus-2 (SARSCoV-2) is first detected in Wuhan, China in December 2019, and th $n$ refined as a novel coronavirus which caused coronavirus disease 2019 worldwide nan $₹ \mathrm{~d}$ is "COVID-19 pandemic". A total of 163.312.429 confirmed cases of COVID-19, includ ng 386.825 virus deaths have been reported worldwide as of $18^{\text {th }}$ May 2021 (1). Vaccinatic $\urcorner$ is the most effective strategy to control the pandemic and COVID-19 vaccines were a ur je it need for this pandemic. The first mass vaccination programme started in eariy December 2020 and Pfizer/BioNTech BNT162B2, Moderna mRNA-1273 and As ${ }^{+}{ }^{+}{ }_{2}{ }^{-}$ner a recombinant adenoviral ChAdOx1-S became first approved COVID-19 vaccines in ine United Kingdom (UK) on 30th of December 2020 (2). Pfizer/BioNTech E VT j 62B2 vaccine was listed for WHO Emergency Use Listing (EUL) on 31 December 2( ``. The SII/Covishield and AstraZeneca recombinant adenoviral ChAdOx1-S were given $\Gamma^{\top} \mathrm{IL}$,n 16 February. The Janssen/Ad26.COV 2.S, the Moderna mRNA-1273 and Thee Sinc . hat . COVID-19 vaccine was listed for EUL on 12 March 2021, 30 April 2021 and 7 May $2 r, L$ respectively (1).

IOVID-19 vaccines are now available in many European countries, the United States (Us, and worldwide. As of 17 May 2021, a total of 1.407.945.776 vaccine doses have been administered worldwide (1). Soon after global use of COVID-19 vaccines, severe allergic 
hypersensitivity reactions to mRNA-based vaccines were reported (2). For example, 11.1 cases of allergic reactions including anaphylaxis occurred per 1 million doses of the Pfizer/BioNTech BNT162B2 COVID-19 vaccination (3) and of 64900 employees who received their first du. 9 of a COVID-19 vaccine including Pfizer/BioNTech BNT162B2 and Moderna mRr.A- '273 vaccines, acute allergic reactions were reported more frequently with the Modarn $n_{n}$ vaccine compared with Pfizer-BioNTech (4). For the Pfizer/BioNTech BNT162B2 COV'L 19 vaccine, $71 \%$ of allergic reactions occurred within $15 \mathrm{~min}$ of vaccination (3). While t'ere are no added

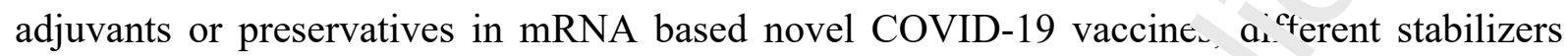
including polyethylene glycol (PEG), polysorbates, tromethamine/ti ${ }^{\prime}$ leta nol were found to be potential to elicit systemic allergic hypersensitivity reactions (5).

Sinovac/CoronaVac COVID-19 vaccine is a 2-dose p-ropiolactone-inactivated, aluminium hydroxide-adjuvanted COVID-19 vaccine authorize by the China National Medical Products Administration on February 6, 2021 (6). Dh.se 3 trial in Brazil including 8,840 participants who received any dose/schedule of inu ac product reported only mild or moderate adverse events (AE) which were most com. 'only pain at the injection site, headache, fatigue, and myalgia. There were few allergic rearlinns, and all were Grade 1 or 2 (6). 260

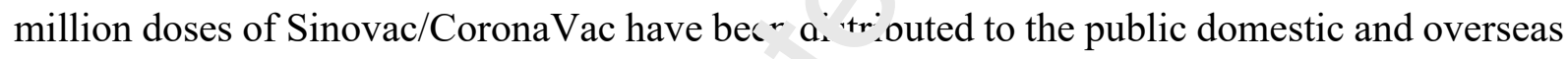
markets for use in adults $\geq 18$ years (6), and ( $O$ 、ID-19 vaccination programme has been started by Sinovac/CoronaVac and Pfizer/BioN $7 \mathrm{e}^{\prime}$, is b YT162B2 vaccines in Turkey on 14th January and 2nd April, respectively. As of 19 n May 2021 total 26.869.851 doses COVID-19 vaccine including mostly Sinovac and ewer Pfizer/BioNTech BNT162B2 vaccines has been administered to healthcare $\mathrm{vo}^{\circ} \mathrm{rrs}^{\mathrm{r}}$ and elderly population (7). In Turkey Phase 3 Sinovac/CoronaVac study ircluding 13,000 healthy participants with the age of 18-59 years, severe adverse events have is t peen reported (6). However, COVID-19 vaccinations including m-RNA based vaccinss rnu Sinovac/CoronaVac seems to be associated with acute allergic reactions. Even tho thh a.iaphylaxis are rare, the other hypersensitivity reactions such as acute eosinophilic pne umnIIIa may be associated with COVID-19 vaccinations. To the best of our knowledge acus eusinophilic pneumonia, rash and dermatitis has not been reported yet after Sinovac/C $\boldsymbol{C} \backslash$ na $\sqrt{ }$ ac vaccination. This paper therefore aims to provide a concise review of the diagno: is anc management of vaccine related acute eosinophilic pneumonia and maculopapular rası inc ish a case presentation. 


\section{Case}

We report a 73-year-old woman who presented to our pulmonology and allergy clinic with maculopapular rash, cough and dyspnea after Sinovac/CoronaVac vaccination. Her v vugh started after first dose of the vaccine and it was an isolated symptom and then mani este $d$ as maculopapular rash and dyspnea after 4th day of second dose of the vaccine. Th.e ${ }^{-e}$ was one month period between first and second dose of the vaccine. Antihistamines we.e no $r$ effective for her rash. The patient denied any allergy, history of allergic disease suck as 'sth na or allergic rhinitis, newly started medication, herbal product use and smoking. She tic not report any constitutional symptoms including weight loss, fever, chronic pain, fat lo $^{\prime}$ e arthralgia, or night sweats. She had hypertension and diabetes history. Her vital signs v. are stable on presentation (Table I). The CBC results indicated eosinophilia (eosinoph a c unt $=600 \mathrm{k} / \mu \mathrm{L}$ ). Further evaluation of the eosinophilia with CT scan could not exclu 2 COVID-19 pneumonia (Figure 1). Because she had dispnea and there were diffuse grou $\mathrm{d} g$ ass densities, consolidation and linear densities in all segments of both lungs. While the S. 'RS-CoV-2 PCR were negative and her anti SARS-Cov-2 anti-spike antibody level wer $\doteq \mathrm{p}_{c}$-itive at the effective level, favipiravir treatment was started. During the evaluation pr $\mathbf{r}^{\mathrm{r}, \boldsymbol{d}}$, she had another negative PCR test for SARS-CoV-2. After third day of favipiravir tre ${ }^{+}$ment the oxygen saturation was $87 \%$ at room level and oxygen treatment were started. B⿺ nd zosinophile count has increased to $2300 \mathrm{k} / \mu \mathrm{L}$. Fiberoptic bronchoscopy was performed $\urcorner$ d specimens of bronchoalveolar lavage (BAL) fluid obtained from right middle lobe. Mu'tipl $:$ biopsies were obtained from the right lower lobe basal segments. Transbronchial ne dle aspiration (TBNA) was performed from 7(right upper hilar ) and 11 (left hilar) node $s^{t}$ at i uns by using EBUS. Skin biopsy was also obtained. The findings of BAL were as follows: macrophage $42 \%$ (normal $>90 \%$ ), lymphocytes $11 \%$ (normal $<5 \%$ ), neutrorhii. $11 \%$ (normal $<5 \%$ ), and eosinophils 36\% (normal $<1 \%$ ). Eosinophile infiltration also detec ed $\mathrm{h}$ lung tissues (Figure 2). There was no granulome, malignite or eosinophile infiltra ion $\mathrm{n}$ lymph nodes. Skin biopsy revealed edema of the superficial dermis and a dense inf ${ }^{1+}$ al 2 of lymphocytes which was found to be associated with drug induced (vaccine) dei nat is. $1 \mathrm{mg} / \mathrm{kg}$ methylprednisolone therapy was started. After seven days of this treatment, her ash resolved with marked improvement of the dyspnea. Thereafter, the patient was cc ntin ed on treatment with oral methylprednisolone (40 mg/day), the dose was planned graa $a_{1}: y$ to be tapered after a period of 4 weeks. A follow-up chest X-ray revealed marked $\mathrm{im}_{\mathrm{r}}$-ovement and total $\mathrm{Ig}$ E decreased from $9662 \mathrm{IU} / \mathrm{ml}$ to $2000 \mathrm{IU} / \mathrm{ml}$. Eeosinophile levels 
was detected at the normal range. She is still on treatment and on the follow up period we plan to continue steroid treatment at least 3 months.

\section{Discussion}

Eosinophilic pneumonia advers reaction (AEs) after vaccination has $r_{c}{ }^{*} e_{1}$, oeen reported. Only two cases have been reported following influenza and pneumococr.-1 vaucination until today. To the best of our knowledge, this is the first report of acute eosino and maculopapuler rash developed after Sinovac/CoronaVac vaccin^tic ? Edsed on 35.8 million doses distributed in Chine, 49 serious AEs reported, including a. apilylaxis, HenochSchonlein purpura, laryngeal odema, demyelination, cerebral hemo thase (6). Based on 17 million doses distributed in Brazil/Indonesia, 162 serious $\mathrm{AF} ; \mathrm{e}_{\mu}$ urted, including fever, dyspnea, death, and headache (6). Based on 3.7 million doses disı outed in Chile, 90 serious AEs reported including anaphylaxis with the rate of $1.7 / 100 . \iota^{\imath} 0$ doses (6). While there were gaps in the detection of rare adverse events especially in $1 \mathrm{~d} \mathrm{f}_{\mathrm{C}}$ adults, there were no reported acute eosinophilic pneumonia and maculopapuler $\mathrm{r}_{\boldsymbol{c}}{ }^{\mathrm{h}} \mathrm{h}$ after Sinovac/CoronaVac vaccination.

Sinovac/CoronaVac COVID-19 vaccine i, a a aminium hydroxide-adjuvanted COVID vaccine (6). Aluminum-containing compoun _. pu marily aluminum hydroxide (AH), have been widely used as adjuvants in the numbe of , ther vaccines such as hepatitis A, hepatitis B, diphtheria-tetanus-containing vaccines, $H_{\lrcorner} \in$ nophilus influenzae type $b$, and pneumococcal vaccines (8). Immunization with alum nur adjuvants induces a Th2 type cell mediated immune response which plays an active role in evelopment and differentiation of eosinophiles after the release of several cytokines in 1 .a.. $\mathrm{g}$ interleukin-3 (IL-3), granulocyte-macrophage colonystimulating factor (GM-C $\mathrm{C}^{-}$), and interleukin-5 (IL-5) (8). Aluminum adjuvant-containing vaccines do not activate $\operatorname{Tr} t$; cells to control strong Th2-mediated immune responses (8). Therefore, aluminum $\mathrm{aa}_{\mathrm{j}} \cdot \mathrm{Va}_{\mathbf{u}}$ ts can induce the production of eosinophils and eosinophilia which may cause e sin`philic pneumonia or dermatitis after vaccination.

In the i. ramure, two cases of eosinophilic pneumonia have been reported following influenza $\cdots$ rneumococcal vaccination. First case is 86-year-old Thai man with severe COPD pres $n$ ted with eossinophilic pneumonia after seven days of inactivated influenza vac ne Vdxigrip, Sanofi Pasteur) injection (9). Second case is A 68-year-old Japanese woman f war ted with eossinophilic pneumonia which developed two days after she received her second vaccination with PPV23 (Pneumovax ${ }^{\circledR}$ NP) (10). Our patients was a 73-year-old woman who presented with maculopapular rash, cough and dyspnea after 4th day of second 
dose of the Sinovac/CoronaVac vaccine. Seasonal influenza (except Fluad) and PPV23 are adjuvant-free vaccines suggesting that the vaccination-associated eosinophilia in previous cases were not caused by aluminum adjuvants. In these patients without any adjuvants an $\mathrm{st}_{\mathrm{L}} \cdot \mathbf{r}$ pathway may lead to eosinophilia. However, in our case hypersensitivity syndrome ar soc aled with eosinophilic infiltration of the lung tissue could be related with aluminum adj"wa.t ${ }^{+}$. Older age and repeated vaccine injections may increase the risk of hypersensitivity $\mathrm{r}$ - ctions and COVID-19 vaccines may also be more prone to allergic or hypersensitivity re cricus. However, in cases of drug-induced eosinophilic pneumonia reported in the litera 'urc, skin eruption is never reported which is an important part of the clinical picture of 1 גr c vse. Skin rash was suggestive of DRESS (The Drug Reaction with Eosinophilia and $S_{\text {, }}$ temic Symptom) and features of the case were a delayed onset. However, the diagne sis of DRESS is challenging because the pattern of cutaneous eruption and the types of orrans $11^{-}$olved are various. In our case, organ involvement including kidney, hearth and livf., ${ }^{1} y_{2}$. phadenopathy and fever was not detected. There was an lung involvement manifester as an eosinophilic pneumonia. We did not do a patch test with diluted vaccine which cor ${ }^{1} \gamma$ better clarify the pathogenesis of the disease.It was our limitation. We used the RegiSC $A_{\mathbf{L}}$ '“ 'scoring system which was published to classify the cases with DRESS reported in the ${ }^{1 * t \epsilon .}{ }^{\text {th }}$,re (9) and our case had less likely DRESS when we used this scoring system. A clinic $\_1$ tı meworkis given in Table 2 .

This immunologic reaction seems $\sim^{1} / \mathrm{e}$ reversible and previous case results indicate that Th2-mediated immune responses is At- - asing with steroid treatment and do not relapse over time. However, there may be a mai, otween repeated aluminum adjuvants exposure and acute eosinophilic pneumonia fre 'sency. A proven diagnosis of hypersensitivity to a vaccine component could be diffirilt. Lowever, aluminum-containing vaccines such as Prevenar 13 should be avoided in patie. ${ }^{+c}$ who had a history of hypersensitivity reactions to any aluminumcontaining vaccines. $\mathrm{h}_{\mathrm{n}}$-ed on the experience with other case reports, patients whose symptoms fully resolve after $s_{\imath} \cdot r_{d} d$ treatment should be under treatment at least 3 months and should be followed up at least one year for the relapse $(10,11)$. Systemic corticosteroids also have been accepted as i. a gold standard treatment for clinical symptoms of DRESS too. Systemic corticoster ids is recomended to be tapered over 6 and 8 weeks to prevent the relapse of various syr'ntur is of this syndrome and to be administered for 2 and 3 months (12)

In conclusion, diagnosing and treating patients who had hypersensitivity reactions after COVID-19 vaccines is challenging and there are still unanswered questions about the long term 
adverse effects of the COVID-19 vaccines. Adjuvants and stabilizers such as polyethylene glycol (PEG) and aluminum seems to lead to allergic and hypersensitivity reactions. Pandemic is urgent and we need to continue vaccination. In the upcoming years, more data will be :01 $\bullet$ available to assess the incidence of different and rare hypersensitivity reactions rela ceu with various types of COVID-19 vaccines and then we may have a new perspective a hou' the risk factors link with vaccine hypersensitivity reactions.

\section{References:.}

1- World Health Organization. 2021, 18 May. Available from: $\underline{h_{2}{ }^{n}}$ s../covid19.who.int/

2- Sokolowska M, Eiwegger T, Ollert M, Torres MJ, Barber Z Lul Giacco S, Jutel M, Nadeau KC, Palomares O, Rabin RL, Riggioni C, Vieths \& A gache I, Shamji MH. EAACI statement on the diagnosis, management and reveni on of severe allergic reactions to COVID-19 vaccines. Allergy. 2021:10.11, 1/all.14739.

3- Center for Disease Control and Prevention. All rg c R r actions Including Anaphylaxis AfterReceipt of the First Dose of Pfizer-BioNT $\sim^{h}$ LUVID-19 Vaccine - United States, December14-23, 2020. https://www.cdc.gov/mmwr/volumes/70/wr. ${ }^{7}{ }^{7}{ }^{7002 \mathrm{e} 1 . h t m ~ A c c e s s e d ~ 07.01 .2020 . ~}$

4- Blumenthal KG, Robinson LB, Camargo $\wedge$, Shenoy ES, Banerji A, Landman AB, Wickner P. Acute Allergic Reactions to 1.'N A COVID-19 Vaccines. JAMA 2021;325(15):1562-1565.

5- Cabanillas B, Novak N. Allergy to $\mathrm{OV}$ D-19 vaccines: A current update. Allergol Int. 2021r 23:S1323-8930(21)000:- y.

6- Evidence Assessment: Sinovac/C $\mathrm{O}^{\mathrm{r}}$ aVac COVID-19 vaccine. FOR RECOMMENDATION BY T HE STRATEGIC ADVISORY GROUP OF EXPERTS (SAGE). April 2021. Avail vie in om: https://cdn.who.int/media/docs/default-

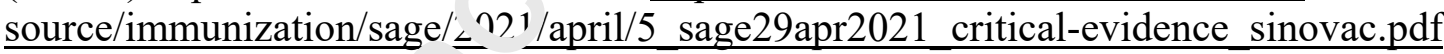

7- TC Sağlık Bakanlığı. A a' ‘a' 'le from: https://covid19asi.saglik.gov.tr/

8- Terhune TD, Deth RC. A ...ninum Adjuvant-Containing Vaccines in the Context of the Hygiene Hypothe ' ${ }^{\mathrm{s}:}$ : A Risk Factor for Eosinophilia and Allergy in a Genetically Susceptible Subp puı tion? Int J Environ Res Public Health. 2018;15(5):901. doi: 10.3390/ijerph1:00ว? 01.

9- Kardaun SH, ₹ idoruff A, Valeyrie-Allanore L, et al. Variability in the clinical pattern of cutaneou, side-effects of drugs with systemic symptoms: does a DRESS syndrome really exj + ? $2:$ J Dermatol. 2007;156:609-611.

10- Pornsuri, sa : P, Suwatanapongched T, Klaewsongkram J, Buranapraditkun S, Rotjanap. $\cap$ P. Acute respiratory failure secondary to eosinophilic pneumonia follnv1.-o influenza vaccination in an elderly man with chronic obstructive pulmonary di sase. Int J Infect Dis. 2014;26:14-6. doi: 10.1016/j.ijid.2014.04.019.

11- n 'uchi R, Iwai Y, Watanabe Y, Nakamura H, Aoshiba K. Acute respiratory failure c 'If to eosinophilic pneumonia following pneumococcal vaccination. Hum Vaccin inmunother. 2019;15(12):2914-2916.

12. Shiohara T, Mizukawa Y. Drug-induced hypersensitivity syndrome (DiHS)/drug reaction with eosinophilia and systemic symptoms (DRESS): An update in 2019. Allergol Int. 20191;68(3):301-308. 


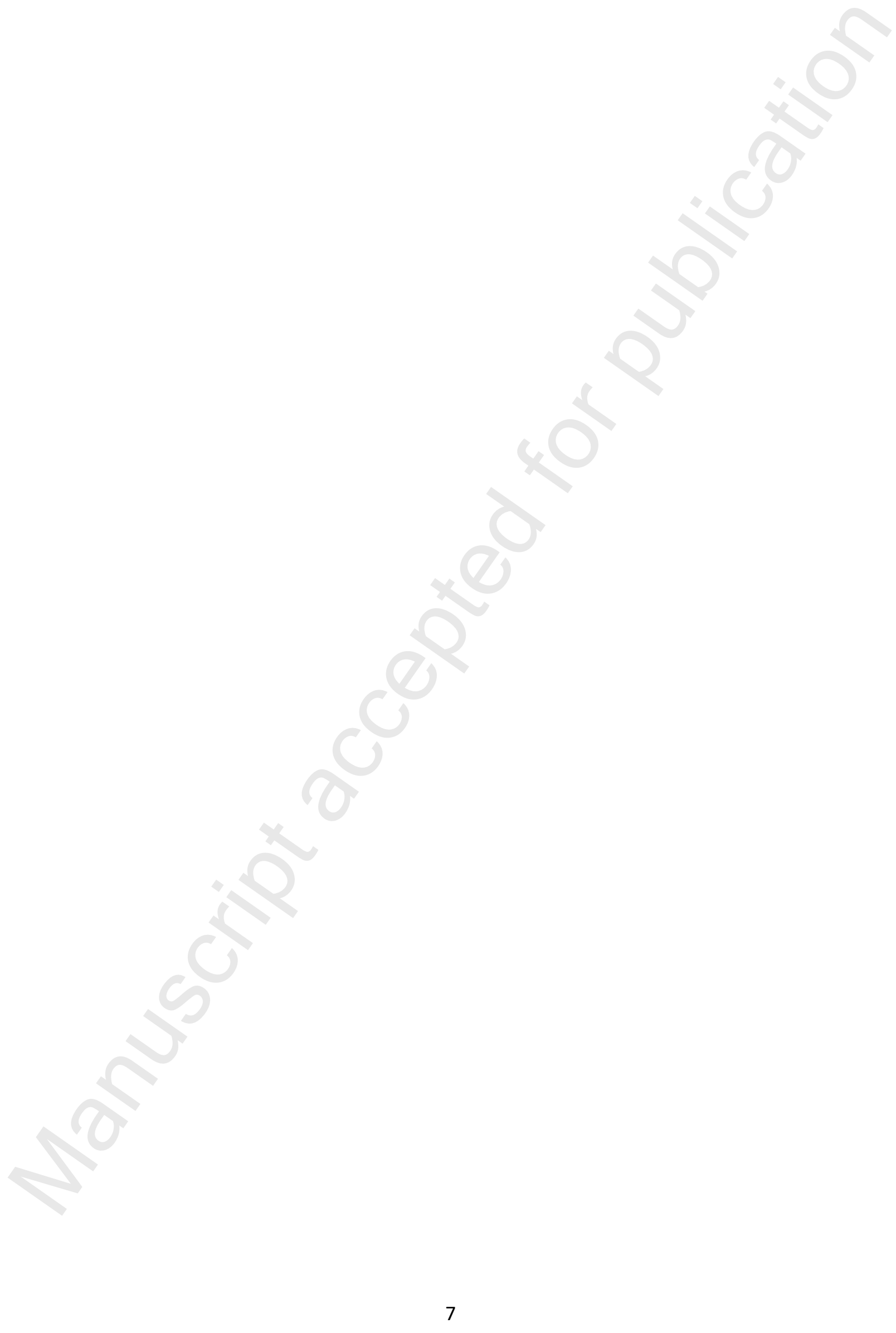

